Documentation et bibliothèques

DOCUMENTATION BIBLIOTHEQUES

\title{
Les premiers sentiers du savoir
}

Aux origines de la bibliothèque du Collège de

Sainte-Anne-de-la-Pocatière (1827-1900)

\section{The First Pathways to Knowledge}

The Origins of the Library of the Collège de

Sainte-Anne-dle-la-Pocatière (1827-1900)

\section{Los primeros senderos del conocimiento}

Orígenes de la biblioteca del Colegio de

Sainte-Anne-de-la-Pocatière (1827-1900)

\section{Gilles Gallichan}

Volume 58, numéro 2, avril-juin 2012

URI : https://id.erudit.org/iderudit/1028907ar

DOI : https://doi.org/10.7202/1028907ar

Aller au sommaire du numéro

\section{Éditeur(s)}

Association pour l'avancement des sciences et des techniques de la documentation (ASTED)

\section{ISSN}

0315-2340 (imprimé)

2291-8949 (numérique)

Découvrir la revue

Citer cet article

Gallichan, G. (2012). Les premiers sentiers du savoir : aux origines de la bibliothèque du Collège de Sainte-Anne-de-la-Pocatière (1827-1900).

Documentation et bibliothèques, 58(2), 53-61. https://doi.org/10.7202/1028907ar
Résumé de l'article

Situé à 100 km à l'est de Québec et fondé en 1827, le Collège de Sainte-Anne-de-la Pocatière est l'un des plus anciens établissements d'enseignement secondaire du Québec. L'article donne un aperçu des origines de la bibliothèque du Collège et de son évolution jusqu'à la fin du XIX ${ }^{\mathrm{e}}$ siècle. Les fondateurs du Collège ont apporté une attention particulière à l'outil pédagogique que représentait déjà la bibliothèque collégiale à cette époque. L'auteur souhaite que l'histoire des bibliothèques des maisons d'enseignement québécoises soit mieux connue et davantage valorisée.
Tous droits réservés (C) Association pour l'avancement des sciences et des techniques de la documentation (ASTED), 2012
Ce document est protégé par la loi sur le droit d'auteur. L'utilisation des services d'Érudit (y compris la reproduction) est assujettie à sa politique d'utilisation que vous pouvez consulter en ligne.

https://apropos.erudit.org/fr/usagers/politique-dutilisation/ 


\section{Les premiers sentiers du savoir ${ }^{*}$ Aux origines de la bibliothèque du Collège de Sainte-Anne-de- la-Pocatière (1827-1900)}

\section{Gilles GallichaN}

gilles.gallichan@videotron.ca
Une bibliothèque est le carrefour de tous les rêves de l'humanité. - Julien Green

\section{RÉSUMÉ | ABSTRACTS | RESUMEN}

Situé à $100 \mathrm{~km}$ à l'est de Québec et fondé en 1827, le Collège de Sainte-Anne-de-la Pocatière est l'un des plus anciens établissements d'enseignement secondaire du Québec. L'article donne un aperçu des origines de la bibliothèque du Collège et de son évolution jusqu'à la fin du XIX ${ }^{e}$ siècle. Les fondateurs du Collège ont apporté une attention particulière à l'outil pédagogique que représentait déjà la bibliothèque collégiale à cette époque. Lauteur souhaite que l'histoire des bibliothèques des maisons d'enseignement québécoises soit mieux connue et davantage valorisée.

The First Pathways to Knowledge: The Origins of the Library of the Collège de Sainte-Anne-de-la-Pocatière (1827-19oo $)^{* *}$

Located $100 \mathrm{~km}$ east of Québec City and founded in 1827, the Collège de Sainte-Anne-de-la-Pocatière is one of the oldest secondary schools in Québec. This article describes the origins of the College's library and its history up to the end of the $19^{\text {th }}$ century. During this period, the founding fathers of the College paid special attention to the pedagogical value of the library. The author wishes to highlight the rich history of school libraries in Québec.

\section{Los primeros senderos del conocimiento Orígenes de la biblioteca del Colegio de Sainte-Anne-de-la-Pocatière (1827-19oo) ${ }^{* * *}$}

Ubicado 100 kilómetros al este de Quebec y fundado en 1827 , el Colegio de Sainte-Anne-de-la-Pocatière es uno de los establecimientos más antiguos de enseñanza secundaria de Quebec. Este artículo ofrece una perspectiva de los origenes de la biblioteca del colegio $y$ de su evolución hasta fines del siglo XIX. Los fundadores del colegio prestaron especial atención a la herramienta pedagógica que representaba la biblioteca académica de esa época. El autor desea que la historia de las bibliotecas de los establecimientos de enseñanza quebequenses se conozca y se valorice más en profundidad.

* Ce titre fait écho au manuel sur le travail en bibliothèque, intitulé: Les chemins du savoir, rédigé par Marcel Mignault en 1974. Ce dernier a été bibliothécaire du Collège de Sainte-Anne-de-la-Pocatière pendant toute sa carrière professionnelle. Ce travail lui est dédié ainsi qu'à tous ceux et celles qui ont œuvré à la bibliothèque et aux archives du collège, préservant ainsi sa mémoire depuis près de deux siècles.

** This article recalls an earlier publication on the work in libraries: Les chemins du savoir by Marcel Mignault published in 1974. Mr. Mignault spent his entire career as the librarian of the Collège de Sainte-Annede-la-Pocatière. This article is dedicated to him as well as all the other members of the personnel of the library and archives who worked to preserve the collections for nearly two centuries.

*** El titulo hace referencia al manual de trabajo en biblioteca, llamado: Les chemins du savoir (Los senderos del conocimiento), escrito por Marcel Mignault en 1974. Mignault fue bibliotecario del Colegio de SainteAnne-de-la-Pocatière durante toda su carrera profesional. Le dedicamos este trabajo a él y a todos aquellos que trabajaron en la biblioteca y en los archivos del colegio, preservando su memoria después de casi dos siglos.
L

E Collège de SAInte-Anne-de-LA-Pocatière, fondé en 1827 par Charles-François Painchaud, est le plus ancien établissement d'enseignement supérieur à l'est de Québec. Il a donné naissance à l'École d'agriculture en 1859, au cégep de La Pocatière en 1968, et il demeure toujours une très importante maison d'éducation secondaire de la Côte-du-Sud et du BasSaint-Laurent ${ }^{1}$. Pour le milieu de la documentation au Québec, La Pocatière représente aussi un centre qui a formé les nombreux techniciens de bibliothèques qui y ont fait des stages entre 1964 et $1971^{2}$.

Si le collège de Sainte-Anne-de-la-Pocatière a été le sujet de plusieurs monographies historiques, sa bibliothèque et ses collections ont été peu étudiées. On sait que l'édifice a subi en décembre 1920 un grave incendie qui a détruit 40000 volumes de la bibliothèque, emportant les collections initiales quavait rassemblées le Collège depuis sa fondation. Heureusement, les archives de l'institution furent préservées et sont toujours conservées aux Archives de la Côte-du-Sud et du Collège de Sainte-Anne ${ }^{3}$. On y trouve les traces des origines d'une bibliothèque collégiale de langue française créée au cœur du Bas-Canada rural au milieu du $\mathrm{XIX}^{\mathrm{e}}$ siècle.

Dans les archives institutionnelles québécoises, un vaste corpus encore peu exploité s'offre à de futures études sur les origines et l'évolution des bibliothèques collégiales ou sur l'instruction publique. À elle seule, l'histoire de la bibliothèque du Collège de Sainte-Annede-la-Pocatière pourrait constituer un chantier de recherche, tant semblent importants le potentiel de ses sources et le rayonnement régional, voire national, de cette institution. Nous limiterons le propos du présent article aux débuts de l'établissement et aux premières décennies d'existence de la bibliothèque, c'est-à-dire jusqu'à la fin du XIX ${ }^{\mathrm{e}}$ siècle. Ce rapide survol ne cherche

1. Pour plus de détails sur le Collège de Sainte-Anne de La Pocatière voir le site : <http ://leadercsa.com/index.php〉.

2. Raymond Boucher, Nicole Lavigne et Alain Boucher, «Les stages de La Pocatière ", dans: Georges-A. Chartrand [dir.], Livre, bibliothèque et culture québécoise. Mélanges offerts à Edmond Desrochers s.j., Montréal, ASTED, 1977, tome 2, p. 577-588.

3. Voir: <www.shcds.org>. L'auteur remercie MM. François Taillon et Michel Dumais pour leur généreuse assistance au cours de cette recherche. 
Au conquérant qui justifiait sa domination coloniale par l'ignorance et l'incompétence des Canadiens français, les collèges $d u$ $X I X^{e}$ siècle répondaient en formant les premières cohortes d'une élite éduquée.

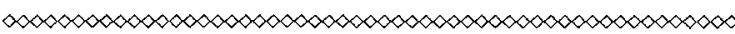

qu'à ouvrir une piste vers l'histoire des bibliothèques des grandes maisons d'enseignement, des couvents, des séminaires et des collèges classiques. Malgré de faibles moyens et des ressources limitées, ces établissements ont défini des rapports sociaux et des référents culturels; ils ont formé des générations d'étudiants et les ont guidés sur "les chemins du savoir». Au conquérant qui justifiait sa domination coloniale par l'ignorance et l'incompétence des Canadiens français, les collèges du XIX ${ }^{e}$ siècle répondaient en formant les premières cohortes d'une élite éduquée, capable de donner au Québec «une histoire et une littérature».

Bien peu des anciens collèges classiques, la plupart devenus des cégeps au cours de la réforme scolaire des années 1960, ont conservé les traces de ce que furent leurs bibliothèques au cours des années antérieures. Les nécessités et les temps nouveaux ont décimé les collections et parfois aussi les archives, les catalogues et les index témoignant de leur importance et de leur rayonnement au cours de l'époque dite de "grande noirceur $»$.

\section{Les origines du Collège de Sainte-Anne}

La fondation du Collège de Sainte-Anne appartient à une période que l'historien des collèges classiques, Claude Galarneau, a appelé « les temps héroïques des fondations canadiennes ${ }^{4}$ ». Une époque difficile pendant laquelle la "nation canadienne" vécut une grande effervescence politique et culturelle ${ }^{5}$. Au milieu des années 1820, le Collège est né d'un besoin du milieu régional et d'une compétition entre trois acteurs : l'Institution royale, les notables laïques de Kamouraska et le clergé catholique.

L'Institution royale, créée en 1801 par l'administration britannique, cherchait à s'implanter dans les régions ${ }^{6}$. Elle ouvrait des écoles et offrait des avantages, mais cependant, comme elle était destinée à assimiler les Canadiens et à les attirer dans le giron

4. Claude Galarneau, Les collèges classiques au Canada français (1620-1970), Montréal, Fides, 1978, p. 16

5. Voir : Maurice Lemire [dir], La vie littéraire au Québec, tome II, 18061839. Le projet national des Canadiens, Sainte-Foy, Presses de l'Université Laval, 1992, XX, $587 \mathrm{p}$.

6. Sur l'Institution royale, voir : Louis-Philippe Audet, Le système scolaire de la province de Québec, Tome III, LInstitution royale, les débuts (18011825), Québec, Presses de l'Université Laval, 1952, xxvi, 323 p. de l'Église officielle anglicane, elle rencontrait la résistance de l'Église catholique ${ }^{7}$ et des Canadiens patriotes. De leur côté, les notables de Kamouraska tentaient de s'organiser et demandaient le soutien de la Chambre d'assemblée du Bas-Canada pour ouvrir une maison d'enseignement secondaire. Ce projet présenté majoritairement par des laïcs inquiétait fort les ecclésiastiques qui craignaient de voir l'instruction publique échapper à la direction et au contrôle de l'Église. L'évêque de Québec, $M^{\mathrm{gr}}$ Bernard Panet, ancien curé de RivièreOuelle qui connaissait bien la Côte-du-Sud, aurait opté de préférence pour des petites écoles de paroisse dans la région. Néanmoins, il donna son accord à l'abbé Charles-François Painchaud, curé de Sainte-Annede-la-Pocatière, qui, sans attendre, avait entrepris la construction d'un collège dans le but d'imposer son projet à tous les intervenants ${ }^{8}$.

Painchaud prend ses concurrents de vitesse et mobilise plusieurs paroisses de la région, dont la paroisse voisine de Saint-Roch-des-Aulnaies. Il organise une souscription publique ${ }^{9}$ et il érige en deux ans un édifice en pierre de quatre étages, mesurant 106 pieds sur 46 ( 35 mètres sur 15$)^{10}$ dans le but « de sortir le monde [...] du sommeil de l'ignorance dans laquelle il nous semble avoir croupi si longtemps ${ }^{11}$ ". L'initiative ne fit pas l'affaire de tous et le curé Painchaud dut composer avec "l'opposition de quelques paroisses rivales ${ }^{12}$ » qui n'appréciaient pas ses méthodes.

Il remporte pourtant son pari téméraire. Construit à force de quêtes, de corvées, d'exhortations ferventes et inspirées, le nouvel établissement ouvre ses portes en $1829^{13}$. Painchaud en est le supérieur et la direction des études est d'abord confiée au jeune abbé Étienne

7. Une tentative de créer un comité catholique de l'Instifution royale dans les années 1820 n’a pas levé la méfiance des autorités religieuses catholiques envers ce système d'instruction publique.

8. Il avait été encouragé en ce sens par Jérôme Demers, du Séminaire de Québec. Claude Galarneau, op. cit, p. 22-23; Serge Gagnon, « Painchaud, Charles-François", Dictionnaire biographique du Canado $[D B C]$ en ligne: 〈www.biographi.ca> ; sur les origines du Collège de La Pocatière, on lira aussi : N.-E. Dionne, Vie de C.-F. Painchaud, prêtre, curé, fondateur du Collège Sainte-Anne-de-La-Pocatière, Québec, Léger Brousseau, 1894, 440 p. du même auteur, Sainte-Anne-de-la-Pocatiere 1672-1910, coll. "Galerie historique " II, Québec, Laflamme et Proulx, 1910, p. 61-125. Même si le Collège était un établissement catholique, Painchaud insista pour que l'appartenance religieuse ne soit pas un critère d’admission aux études. Il écarta toute discrimination envers des non-catholiques et on ne tolérait "aucune discussion religieuse [...] contre quelque religion que ce soit $\%$. Manifeste de $M$. Painchaud, $1^{\mathrm{cr}}$ août 1828, cité par Wilfrid Lebon, Histoire du Collège de Sainte-Anne-de-LaPocatière, Le premier demi-siècle 1827-1877, Québec, Charrier \& Dugal, 1948 , p. 363. Mentionnons que, de 1812 à 1824, La Pocatière avait une école de l'Institution royale tenue par Robert Dupont et qui accueillait annuellement 25 élèves, L.P. Audet, Le système scolaire, op. cit. p. 136, 140.

9. Même le gouverneur britannique, sir James Kempt, a soutenu le projet en faisant un don de 25 E. La Gazette de Québec, 22 septembre 1828, p. 2.

10. "Collège de Sainte-Anne ", La Gazette de Québec, 4 décembre 1828, p. 3.

11. "Collège de Sainte-Anne », La Gazette de Québec, 16 juillet 1827, p. 2.

12. Ibid.

13. Le village avait précédemment une ancienne école où furent donnés les premiers cours de latin en 1827 et 1828 . Cette maison, qui était probablement l'école de l'Institution royale, se trouvait sur le site de I'École d'agriculture de La Pocatière, devenue l'Institut de technologie agroalimentaire (ITA). L'ancienne maison fut abandonnée en 1829 au 


\section{Figure 1}

Le premier collège de Sainte-Anne-de-la-Pocatière (1829-1842).

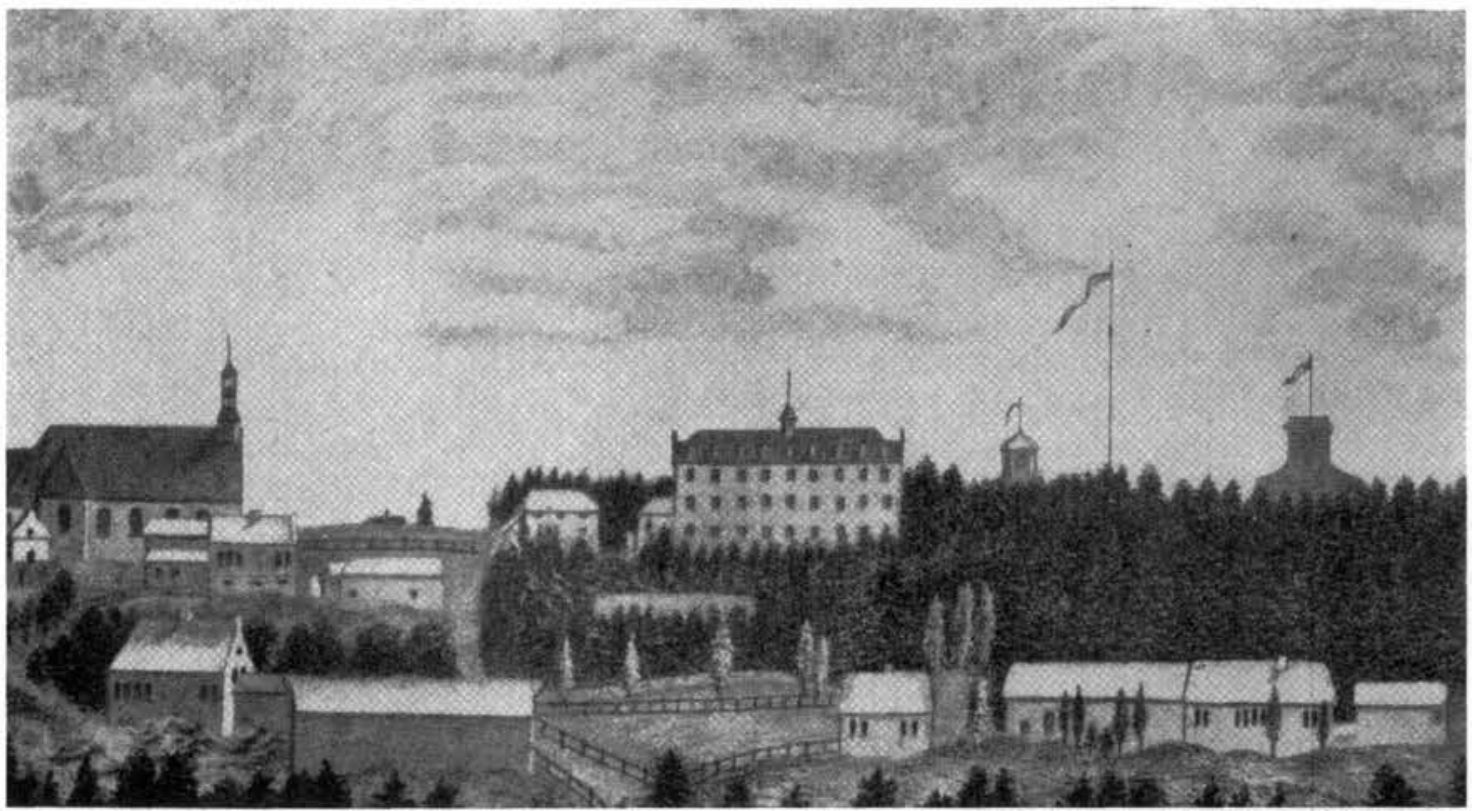

(Archives du Collège de Sainte-Anne-de-la-Pocatière)

Chartier (1798-1853) reconnu pour ses idées politiques libérales et patriotes ${ }^{14}$. Pour pallier le manque de professeurs, on adopte le système d'enseignement du pédagogue anglais Joseph Lancaster, basé sur l'entraide mutuelle entre les élèves ${ }^{15}$. On recrute aussi des finissants séminaristes de Québec pour y enseigner pendant un an ou deux.

La première cohorte, celle de 1829-1830, compte 20 pensionnaires et une dizaine d'externes. Le nombre d'élèves passera à 70 en 1834 et doublera le cap de la centaine à la fin de la décennie 1830. Pendant ces années, le programme d'études est complété et le cours est offert à tous les niveaux à partir de 1834 .

Les méthodes progressistes du directeur Chartier sont bientôt remises en question et jugées trop permissives. En 1830, il est remplacé par Alexis Mailloux, lequel impose une discipline plus stricte. Dans une maison d'enseignement, il fallait nécessairement des livres et des manuels. Le manque de ressources oblige le fondateur et les premiers enseignants à solliciter des dons de livres et à user d'expédients pour en acquérir. Mais l'importance de doter le collège d'une bibliothèque comptant "de bons auteurs modernes ${ }^{16}$ "

profit du nouveau collège. Voir : La Gazette de Québec, 4 décembre 1828 , p. 3 .

14. Gilles Boileau, Etienne Chartier: La colère et le chagrin d'un curé patriote, Québec, Septentrion, 2010, 360 p. Le discours d'inauguration du collège prononcé par l'abbé Chartier était politiquement très engagé et souleva un tollé dans les milieux conservateurs britanniques du Bas-Canada.

15. Wilfrid Lebon, Histoire du College de Sainte-Anne, op. cit, p. 14 et p. 364.

16. C.-F. Painchaud, Manifeste (1 $1^{\text {er }}$ août 1828 ), loc. cit. p. 366. n'échappait pas à ces hommes qui avaient étudié dans les séminaires à Québec, à Montréal ou à Nicolet, et qui avaient eu accès à cet outil pédagogique essentiel. L'abbé Painchaud va aussi rapidement investir dans l'équipement d'un premier cabinet de physique et confier l'enseignement de cette discipline à JosephPierre Maurault (1819-1871) ${ }^{17}$.

Le collège devait fournir des manuels et des dictionnaires à ses élèves en plus de leur offrir matière à lire et à développer leurs connaissances. On sait que dans les écoles, collèges et séminaires québécois du XIX ${ }^{e}$ siècle, les professeurs palliaient le manque de manuels en dictant les leçons aux élèves qui les retranscrivaient studieusement dans leurs cahiers. Cette forme de lien entre l'imprimé et le manuscrit, dont l'origine remonte à l'Antiquité grecque et aux traditions scolastiques du Moyen Âge, s'est perpétuée au Québec et particulièrement dans les milieux éloignés des centres de production de manuels imprimés. Tous les anciens collèges classiques du Québec ont diffusé leur enseignement par le truchement de manuels

17. W. Lebon, op. cit., p. 28-29; Catalogue des anciens élèves du Collège de Sainte-Anne-de-la-Pocatière 1827-1927, Québec, L'Action sociale, 1927, p. 26-27. Le Collège pourra se vanter de la réputation de ses professeurs de physique et de chimie au XIX ${ }^{\mathrm{e}}$ siècle, en particulier Grégoire Tremblay qui enseigne de 1843 à 1854 , Achille Vallée, professeur de 1863 à 1872 et Louis-M. Destroismaisons, de 1897 à 1903 , tous considérés comme des chercheurs et des novateurs dans leur discipline. Archives de la Côte-duSud et du Collège de Sainte-Anne (La Pocatière) [à l'avenir ACSCSA], F $100 / 163 / 77$ Registre 100.3, p. 173-175. 
retranscrits à la main ${ }^{18}$. Pour les élèves, la bibliothèque prenait une valeur encore plus grande du fait que le livre imprimé était une denrée rare et précieuse.

Painchaud affirmait vouloir cultiver le talent des élèves "en leur faisant aimer l'étude et en les y attachant par toutes sortes d'encouragements et l'éloignement de tout désagrément inutile 19 ". À ses yeux, outre le programme établi, les élèves devaient développer leurs connaissances dans les sciences de la nature, la géographie, l'astronomie, la navigation, l'architecture, le dessin, la peinture, l'histoire générale et nationale, la politique ainsi que dans "notre constitution et les lois principales qui nous régissent et dont l'ignorance fait tant de $\mathrm{mal}^{20}$ ".

Les premières acquisitions de la bibliothèque se sont faites essentiellement par dons et par legs testamentaires. Puis, malgré des budgets extrêmement modestes, quelques subventions ont permis de doter l'établissement d'accessoires divers, d'appareils scientifiques et de la documentation pertinente aux études classiques. $\mathrm{Ce}$ fut notamment le cas des livres et autres fournitures que l'abbé Jean Holmes rapporta de son voyage en Europe en 1836-1837. Ce furent là les premiers élans donnés à la bibliothèque du Collège.

\section{Les donations}

Le premier legs important venu enrichir la bibliothèque du Collège fut celui du fondateur, CharlesFrançois Painchaud qui, par son testament rédigé en 1837, cédait tous ses biens au collège. À sa mort en février 1838, sa bibliothèque de 566 volumes fut intégrée au fonds initial de l'établissement ${ }^{21}$. Quelques mois plus tard, la succession du curé de Rivière-Ouelle, l'abbé Louis-Marie Cadieux (1785-1838), cédait une collection de 550 volumes $^{22}$. L'année suivante, la bibliothèque du curé de Saint-Roch-des-Aulnaies, l'abbé Louis Brodeur (1776-1839), fut intégrée à celle du collège ${ }^{23}$. En 1843, le curé de Beaumont, Louis Raby, léguait sa collection de 870 volumes, considérée particulièrement précieuse en histoire et en littérature ${ }^{24}$. Le collège reçut aussi en 1847 une partie de la bibliothèque de l'abbé Urbain Orfroy (1766-1846), un des prêtres français émigrés de la Révolution française, arrivé à Québec en 1796. Ami

18. Paul Aubin, "Les manuels scolaires en Nouvelle-France, dans la "Province de Québec» et au Bas-Canada", dans: P. Fleming, G. Gallichan, Y. Lamonde [dir.], Histoire du livre et de l'imprimé au Canada. Vol. 1 Des débuts à 1840 , Montréal, Presses de l'Université de Montréal, 2004, p. 271-274.

19. C.-F. Painchaud, Manifeste ( $1^{\text {er }}$ août 1828), loc. cit. p. 364.

20. Ibid. p. 366.

21. Catalogue de la bibliothèque de $M$. Painchaud, ACSCSA, Fonds Painchaud, 4-LXXVI; Testament de Ch.-François Painchaud, Fonds du Collège, $\mathrm{F}_{100 / 242 / 46 .}$

22. Catalogue de la bibliothèque du révérend Ls-Marie Cadieux, ACSCSA, Successions, 50-LXXXIX

23. N.-E. Dionne, Vie de C.-F. Painchaud... op. cit. p. 234-235; ACSCSA, F $100 / 242 / 27$

24. WILfRID LeBon, op. cit., p. 59; Catalogue de la bibliothèque de Louis Raby, 11 juillet 1843, ACSCSA, Successions, 50-XCI. de Charles-François Painchaud, il avait activement soutenu son projet de fondation ${ }^{25}$.

Ces premières donations, bientôt suivies par d'autres, ont constitué des apports importants à la collection documentaire du Collège au cours de ses premières années d'existence. À défaut de généreuses fondations et de dons considérables, les legs de successions ecclésiastiques ont assurément été une des bases essentielles de la bibliothèque. Ainsi, par dons, successions et sédimentation documentaire, l'établissement a pu se doter en quelques années d'une bibliothèque importante pour l'époque. Le directeur, l'abbé Alexis Mailloux, tenait à investir dans le développement de la bibliothèque et était convaincu de son importance ${ }^{26}$. Dans un mémoire présenté à la Législature du CanadaUni en 1843 , on apprend que la bibliothèque du collège compte alors 3860 volumes ${ }^{27}$.

\section{Le voyage de l'abbé Holmes}

En mai 1836, l'abbé Jean Holmes (1799-1852), professeur au Séminaire de Québec, entreprend un voyage d'études aux États-Unis et en Europe pour s'informer sur les régimes pédagogiques et sur la formation des maîtres. Il est mandaté par le gouvernement et se charge aussi de commandes diverses d'achat de livres et de matériel pour des maisons d'enseignement. Au moins huit collèges et couvents, dont le Collège de SainteAnne-de-la-Pocatière, l'autorisent à faire des acquisitions pour leurs bibliothèques et leurs laboratoires ${ }^{28}$.

Dès l'année 1836, l'abbé Holmes expédie à SainteAnne des caisses de manuels et de grammaires françaises ainsi que des livres et des appareils de physique et de chimie, des générateurs électriques, des baromètres, thermomètres, télescopes, microscopes et autres instruments optiques destinés à l'enseignement des sciences ${ }^{29}$. Il achète aussi en France de la papeterie - papier, crayons, plumes, encres, étiquettes, etc. - et du matériel de reliure. À Paris, le 30 mars 1837, Holmes s'acquitte d'une commande composée essentiellement de livres destinés à Sainte-Anne-de-La-Pocatière. La facture totalise 2172 francs, une somme importante pour l'époque. Holmes achète chez Hachette, à la librairie Gaume \& frères et chez $\mathrm{A}$. Bourgeois de Paris environ 185 titres, en gros 840 volumes, lesquels sont expédiés au Bas-Canada par «caisse et emballage sous toile $e^{30}$. On y trouve un intéressant échantillon

\footnotetext{
25. Urbain Orfroy, 29 septembre 1847, ACSCSA, Successions, 50-III.

26. Alexis Mailloux, 27 septembre 1841 , ACSCSA, Bibliothèque, $523: 8$.

27. Collège de La Pocatière, Mémoire à la Législature, octobre 1843, ACSCSA, Procure, 43-I.

28. Claude GalarneaU, "Holmes, John », DBC en ligne: www.biographi. $\mathrm{Ca}$

29. ACSCSA, fonds du collège, $\mathrm{F}_{100} / 163 / 78$ et $\mathrm{F} 100 / 173 / 79$

30. [J. Holmes]. Paris. Facture de la caisse S. Ste A. $n^{o} 4$, destinée au Séminaire de $S^{t e}$ Anne, expédiée par Messieurs Gaume frères, 5 rue du Pot-de-fer, Saint-Sulpice, recommandée à $M M$. $W^{m}$ Pemberton et $C^{i e}$, London, et à $M M$. Th Cary, à Québec, [payé à Paris pour le Séminaire de $S^{\text {te }}$ Anne, le 30 mars 1837]. ACSCSA, F 100.
} 


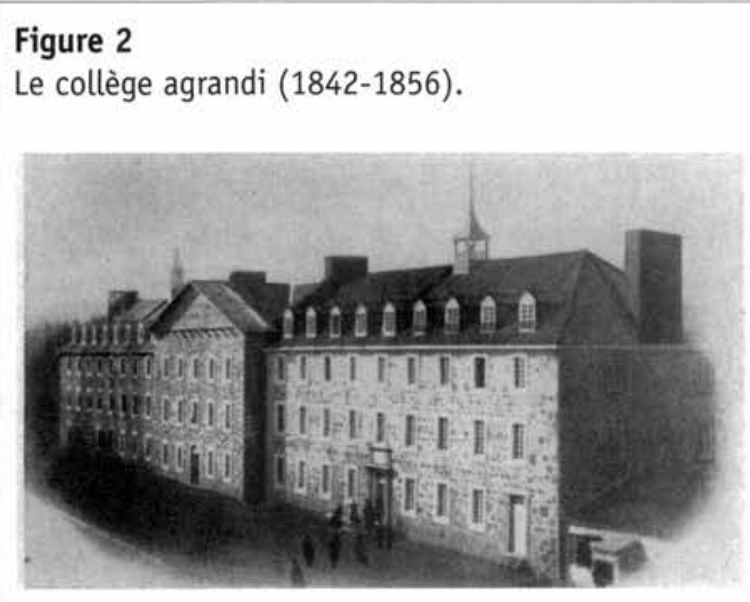

(Archives du Collège de Sainte-Anne-de-la-Pocatière)

de ce que pouvait offrir, dans les années 1830, une bibliothèque collégiale soigneusement choisie pour les élèves avec l'aide des meilleurs libraires français.

Le connaissement de l'envoi fait de Paris par Jean Holmes à Sainte-Anne-de-la-Pocatière nous révèle un choix éclectique de sujets: chimie, astronomie, dessin, architecture, langues, grammaires anciennes et modernes, littérature, philosophie, droit, hagiographie, théologie, histoire et géographie. Plusieurs titres portent sur la découverte du monde, sujet de grand intérêt à l'époque. Peu de littérature contemporaine, sinon des anthologies, mais plusieurs classiques grecs et latins, les Pères de l'Église, et les grands classiques de la littérature française de Molière à Corneille, de Descartes à Bossuet jusqu'à Chateaubriand, un favori de l'abbé Painchaud et, du côté des lettres anglaises, Francis Bacon et John Locke.

Holmes achète aussi des cartes géographiques en français, des tableaux didactiques pour l'enseignement et des modèles pour les exercices de dessin. Les acquisitions de l'abbé Holmes en 1837 ont certainement constitué un accroissement très significatif en quantité et en qualité pour la bibliothèque du Collège. De plus, ces livres étaient tous d'édition récente et assuraient un enseignement de connaissances scientifiques bien à jour.

\section{Initiation à la lecture}

L'abbé Painchaud, le premier supérieur du Collège, était un esprit romantique, profondément marqué par la lecture des œuvres de Chateaubriand. En 1826, il avait même écrit au célèbre ministre et ambassadeur de Charles X pour lui exprimer les transports d'émotion que lui avait inspirés son Génie du christianisme, publié en 1802 . Le vicomte lui avait courtoisement répondu en 1827 que les éloges d'un prêtre canadien le touchaient plus profondément que "les applaudissements d'un prince de l'Église ${ }^{31}$ ". Par cet échange de correspondance, on apprend également que Painchaud était un fervent lecteur des auteurs de la contre-révolution, Louis de Bonald et Joseph de Maistre. On peut donc imaginer que ces auteurs étaient présents dans la bibliothèque du Collège avant même que l'abbé Holmes en expédie de nouvelles éditions en 1837.

$\mathrm{Si}$ on ne dispose pas d'un catalogue complet de la première collection de la bibliothèque, un document d'une très grande valeur, conservé aux Archives de la maison, retrace la circulation des livres prêtés aux élèves pendant l'année scolaire $1839-1840^{32}$. Il s'agit d'un registre où ont été consignés les titres des ouvrages prêtés et le nom des élèves fréquentant la bibliothèque. On y découvre un remarquable échantillon des lectures d'élèves canadiens nés aux environs de 1825 . Les thèmes sont des plus variés: histoire, géographie, littérature, religion, navigation, agriculture, etc. Il serait long et fastidieux de procéder ici à des énumérations d'ouvrages, mais à titre d'exemples en voici quelques-uns : un petit Atlas, l'Histoire de France, un Traité d'agriculture, Euvres de La Bruyère, le Molière des collèges, Essai sur l'éloquence, Le Concile de Trente, Itinéraire de Paris à Jérusalem (Chateaubriand), l'Ami des enfants, $\mathrm{La}$ Découverte de l'Amérique, etc. On y apprend aussi que la lecture faite au réfectoire à ce moment-là était l'Histoire du Canada sous la domination française, de Michel Bibaud, publiée à Montréal en 1837.

\section{Un collège agrandi (1841-1842)}

Pour réagir contre l'Union du Haut et du BasCanada qui visait à marginaliser et à assimiler les Canadiens, Alexis Mailloux et plusieurs notables, dont le seigneur et conseiller législatif Amable Dionne, René-Édouard Caron et le journaliste du Canadien et ancien bibliothécaire parlementaire Étienne Parent, lancent en 1840 une souscription pour la mise sur pied à Sainte-Anne d'un cours commercial bilingue destiné à favoriser la participation des Canadiens au domaine de l'économie et du commerce.

Cet élargissement du programme se traduit par un agrandissement du collège auquel on ajoute une nouvelle aile en $1842^{33}$. Le cours commercial de trois ans peut alors servir de préparation au cours classique. Les élèves qui suivent la totalité de l'enseignement commercial et classique peuvent cumuler neuf années d'études. Le Collège engage donc de nouveaux professeurs et

\footnotetext{
31. ADRIEN GARNIER, "Une lettre de Chateaubriand à un prêtre canadien en 1827 ", L'Action catholique, 6 juin 1932; M. LEMIRE [dir.], La vie littéraire..., op. cit., p. 441-442; WILFRID LEBON, op. cit. 359-362.

32. Catalogue des livres du Collège de Ste-Anne actuellement entre les mains des étudiants, outre les livres proprement classiques, 1839-1840, 103 p. ACSCSA F100/360/1-4.

33. "Collège de Ste-Anne ", Le Canadien, $1^{\text {er }}$ décembre 1841 , p. 2.
} 
plusieurs manuels s'ajoutent pour satisfaire les besoins nouveaux ${ }^{34}$.

La bibliothèque des années 1840 était rassemblée dans une grande pièce du deuxième étage de la maison, appelée la "salle élémentaire », car elle avait précédemment servi de classe pour les éléments latins. HenriRaymond Casgrain (1831-1904), futur prêtre, auteur et critique littéraire qui fit ses études à Sainte-Anne-de-laPocatière entre 1842 et 1852, a laissé dans ses mémoires une description de cette salle :

«Je fais, en passant, mémoire de la salle élémentaire qui me rappelle quelques-unes des meilleures heures de ce temps, heures de silence et d'oubli de toutes choses, absorbé que j'étais dans la lecture et l'annotation des chefs-d'œuvre de l'Antiquité et des lettres modernes. [...] Voici la longue table, au centre, toute garnie de lecteurs penchée sur leurs livres, en face les uns des autres. Plusieurs sont assis sur les bancs en sens inverse à la table, tenant en main revues et journaux dont un grand nombre sont éparpillés tout le long de cette même table ${ }^{35}$."

L'abbé Casgrain nous apprend en outre que la salle était décorée de gravures et de chromolithographies, dont deux portraits remarquables de Napoléon, l'un en premier consul et l'autre en empereur. Ironiquement, on y conservait aussi, pieusement encadrée, la lettre originale que Chateaubriand, génial adversaire et néanmoins admirateur de Napoléon, avait écrite à l'abbé Painchaud en 1827.

En 1847, Célestin Gauvreau succède à Alexis Mailloux comme supérieur du Collège. Il a déjà pour le seconder l'abbé François Pilote (1811-1886) qui enseigne depuis 1836 et qui cumule les postes de directeur, d'administrateur et de procureur de l'établissement. Lorsque Gauvreau doit démissionner pour des raisons de santé en 1853 , François Pilote devient naturellement le nouveau supérieur. Par son travail en faveur de l'agronomie et de la colonisation, il est considéré comme le second fondateur du Collège de Sainte-Anne qu'il marquera profondément de son dynamisme. C'est sous son administration qu'est fondée l'École d'agriculture en 1859 ; il dirigera le Collège jusqu'en 1870.

Pendant toutes ces années, l'abbé Pilote imprime aussi un élan à la bibliothèque et accorde une attention particulière à ses collections. Dès les années 1830 et 1840 , il fait d'importants achats pour lui-même, pour des amis et pour la bibliothèque du Collège. À défaut de librairie locale, il revend à bon prix des livres aux élèves souhaitant s'acheter quelques titres ${ }^{36}$. Il s'approvisionne

34. Wilfrid Lebon détaille les livres à l'usage des élèves du Collège dans les années 1840 , op. cit. p. 57-58, note 13 .

35. Henri-Raymond Casgrain, Souvenances canadiennes, tome 2, texte inédit cité par W. LeBON, op. cit., p. 383.

36. Livres achetés de Mr Comte en 1840 pour le College de Ste Anne. «N.B. Les ouvrages marqués $\mathrm{B}$ sont restés à la bibliothèque, les autres ont été surtout à Québec, chez Thomas Cary, chez Bossange, Morel et $\mathrm{C}^{\mathrm{ie}}$, et auprès de Joseph Comte, procureur pour les Sulpiciens à Montréal ${ }^{37}$. De son côté, l'abbé Holmes continue aussi de fournir François Pilote en livres destinés à la bibliothèque ${ }^{38}$.

Au milieu du siècle, le rayonnement et le prestige $\mathrm{du}$ Collège s'étendent. L'établissement accueille alors plus de 500 élèves et sa bibliothèque s'enrichit. En 1850 , un incendie se déclare au sous-sol du collège. Avec une pompe dérisoire, des volontaires combattent le feu pendant que les élèves procèdent à l'évacuation de la bibliothèque ${ }^{39}$. Heureusement, le feu est circonscrit, mais le désordre général a entraîné des pertes et, au moment de l'inventaire, on ne retrouve pas certains livres ${ }^{40}$.

Le Collège n'acquiert pas les livres uniquement pour l'enseignement, pour les revendre ou pour doter sa bibliothèque. La tradition de distribution aux élèves de livres de prix en fin d'année y est bien implantée. En 1840 , le Collège achète au coût de 10£, 93 volumes destinés aux gagnants de prix d'excellence. Outre les livres religieux, on y recense des éditions de Bossuet, de Fénelon, de Boileau, des histoires édifiantes et même un Code civil de Napoléon ${ }^{41}$. À la fin de chaque année scolaire, c'est une fierté pour un jeune de retourner dans sa famille avec des livres qui représentent les trophées de son application à l'étude. En 1845, Henri-Raymond Casgrain, terminant son année de rhétorique, reçoit le premier prix de déclamation française, ce qui lui vaut une édition bruxelloise en quatre tomes des œuvres de Jean Racine ${ }^{42}$.

Une étude plus complète des archives et un repérage des ex-libris dans les livres du XIX ${ }^{\mathrm{e}}$ siècle conservés dans les collections des bibliothèques au Québec permettraient de mieux documenter et d'échantillonner le catalogue de ces livres de prix distribués dans les collèges. On éclairerait ainsi un réseau non négligeable de la librairie et de la circulation du livre dans le BasCanada en plus de mieux cerner le prestige du livre au sein de la société de l'époque ${ }^{43}$.

revendus aux écoliers \%. ACSCSA 14-VIII.

37. ACSCSA 14-VIII.

38. Fourni à Messire Pilote pour le College de Ste Anne, ACSCSA, 4.3-X ; Québec, 27 juin 1844. Le Collège de Ste Anne. Dû̀ à Messire Holmes, ACSCSA, 112-XLVI.

39. WiLfRID LeBON, op. cit, p. 103.

40. Thomas Chapars à François Pilote, 14 mai 1853, ACSCSA, Bibliothèque - Pilote 20-XCII.

41. Liste des livres envoyés à Messire Pilote pour choisir ceux qui seront propres à être donnés en prix. ACSCSA 14 -VIII.

42. JEAN RaCine, Euvres, Bruxelles, 1843, 4 tomes. Il s'agit d'un bel exemple des contrefaçons belges qui circulaient à l'époque au Bas-Canada. L'ouvrage portant l'ex-libris de la remise des prix de 1845 au Collège de La Pocatière est conservé à la Bibliothèque de l'Université Laval sous la cote PQ 1885/1843/1-4. Je remercie le professeur Jacques Hellemans, de l'Université Libre de Bruxelles pour m'avoir signalé l'existence de cet exemplaire.

43. Pour un aperçu de l'histoire des livres de prix d'excellence, voir François Landry, "Les livres de prix au Québec", dans: Carole Gerson et JaCQues Michon [dir.], Histoire du livre et de l'imprimé au Canada, vol. III De 1918 à 1980, Montréal, Presses de l'Université de Montréal, 2007, p. 88-91. 


\section{Figure 3}

Le collège couronné de son premier dôme (1856-1881).

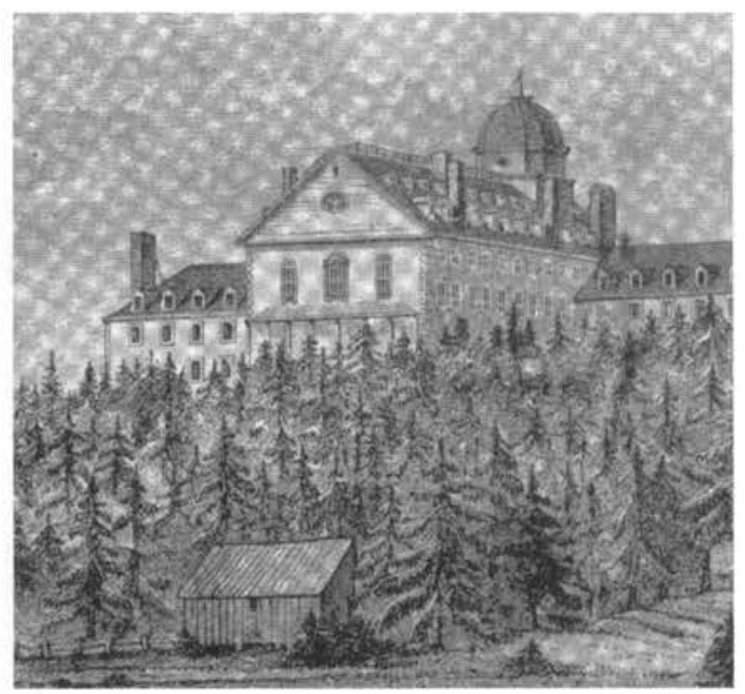

(Archives du Collège de Sainte-Anne-de-la-Pocatière)

\section{Un projet de bibliothèque ecclésiastique (1852-1854)}

En 1852, l'abbé François Pilote propose de créer au Collège une bibliothèque collective de souscription pour les prêtres de la Côte-du-Sud et des paroisses de Kamouraska. Il constatait la difficulté, pour un curé de village souvent peu fortuné, de se constituer une bibliothèque religieuse valable. La bibliothèque ecclésiastique de Sainte-Anne-de-la-Pocatière serait une ressource documentaire collective qui élargirait leurs choix de lectures. Un premier projet pour la «diffusion de la science ecclésiastique " est élaboré en avril 1852 et proposé à une douzaine de prêtres curés des paroisses situées entre L'Islet et Rivière-du-Loup ${ }^{44}$. Une telle collection développerait des thèmes de théologie, d'hagiographie, de pastorale, de catéchèse, d'enseignement religieux et de prédication, basés sur le système de l'encyclopédie théologique de l'abbé Jacques-Paul Migne ${ }^{45}$.

Le projet de l'abbé Pilote vient près de se réaliser en 1854. Moyennant une souscription annuelle de "quatre piastres", chaque membre pourrait emprunter un ou deux volumes du fonds constitué d'environ 300 volumes de la section religieuse de la bibliothèque du Collège, de 100 volumes disponibles de l'encyclopédie Migne et des nouvelles publications ajoutées à la collection par les souscriptions annuelles ou par dons.

44. ACSCSA, 52-L.

45. Célestin Gauvreau à François Pilote, 14 novembre 1854., ACSCSA, CAC 11:49, fonds Gauvreau 11-XLIX. Prospectus d'une bibliothèque ecclésiastique du Collège Ste-Anne à l'usage du clergé des comtés de L'Islet et de Kamouraska. ACSCSA, F10o/360/7 ; publiée dans les années 1840 , l'encyclopédie Migne comptait 171 volumes.
Ce projet de Pilote pour une bibliothèque centrale de théologie à La Pocatière se situe dans le mouvement de renouveau religieux qui touchait la région depuis le passage de $\mathrm{M}^{\mathrm{gr}}$ de Forbin-Janson au Bas-Canada en 1841. Les fidèles appréciaient les retraites prêchées et les prédications qui demandaient de la part des prêtres réflexions, préparations et lectures. L'initiative témoigne aussi du succès des formules de bibliothèques de collectivités, basées sur la coopération et la mise en commun des ressources. Mais, peut-être en raison du manque de souscription ou des distances entre les villages, le projet ne fut pas réalisé.

\section{Progrès des collections collégiales}

En 1855, la bibliothèque du Collège atteint les 6000 livres ${ }^{46}$ et elle en comptera 10 ooo vers 1875 , ce qui en faisait sûrement la plus importante de la région. À cette époque, l'édifice a de nouveau été agrandi. Les plans, dessinés par Thomas Baillairgé, soulignent son ordonnance classique et le coiffent d'un dôme construit en $1856^{47}$. Le Collège développe bientôt, en plus de la bibliothèque générale, une bibliothèque pour les séminaristes et enseignants et une autre pour les élèves, selon un usage répandu dans les établissements secondaires.

L'importance de La Pocatière comme centre régional s'affirme. En 1861, deux ans après la fondation de l'École d'agriculture, les Sœurs de la Charité ouvrent un pensionnat et une école pour jeunes filles de manière à poursuivre l'œuvre des demoiselles Guy qui avaient fondé un refuge pour les orphelins ${ }^{48}$. La même année paraît La Gazette des campagnes, fondée par Firmin Proulx, laquelle sera publiée jusqu'à la fin du siècle. C'est le premier journal d'importance dans la région de la Côte-du-Sud et du Bas-Saint-Laurent et ce n'est pas un hasard si ce journal régional est né dans une ville collégiale. L'idée était celle de François Pilote et plusieurs professeurs du Collège y collaboraient ${ }^{49}$.

Dans les dernières décennies du siècle, le Collège est doté d'un cabinet de physique fort bien équipé, d'un musée d'histoire naturelle, d'une collection lapidaire et d'un musée de numismatique ${ }^{50}$. Sous l'administration du neuvième supérieur, l'abbé Charles Trudelle (de 1878 à 1886), le bâtiment est de nouveau agrandi et un second dôme couronné d'une lanterne est érigé. C'est dans cette partie de l'édifice que la bibliothèque est aménagée en 1881. L'espace est aussi utilisé pour exposer

\footnotetext{
46. Françols Pilote à Jean-Baptiste Meilleur, 20 janvier 1855, ACSCSA, fonds Pilote, 22-LXVI.

47. Pierrette Maurais, La paroisse de Sainte-Anne-de-la-Pocatière, 17151990, La Pocatière, Imprimerie Fortin Itée, 1990, p. 44.

48. Ibid. p. 52-53.

49. ANDRÉ BEAULIEU et JEAN HAMELIN, La presse québécoise des origines à nos jours, tome 2, 1860-1879, Québec, Presses de l'Université Laval, 1975, p. 16-17.

50. Wilfrid Lebon, Histoire du Collège de Sainte-Anne-de-la-Pocatière, tome 2, Le second demi-siècle 1877-1927, Québec, Charrier et Duval, 1949, p. 82 .
} 


\section{Figure 4}

Le collège à la fin du XIXe siècle avec son nouveau dôme qui abritait une partie de la bibliothèque.

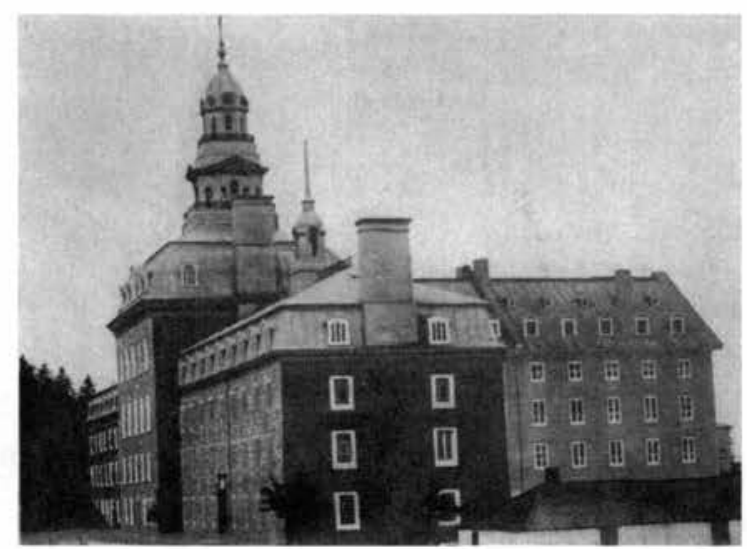

(Archives du Collège de Sainte-Anne-de-la-Pocatière)

les collections de minéraux et de médailles du Collège ainsi qu'une imposante maquette représentant le village en 1867. L'historien du Collège, Wilfrid Lebon, décrit ainsi les lieux :

"Avant la construction du dôme, la bibliothèque était placée au second étage de l'avantcorps, à l'endroit même qui servait jadis de chapelle. Pendant les travaux de l'été 1881, on dut en transporter les volumes un peu partout dans la maison. Il s'agissait de les remettre en place. M. Trudelle avait déjà commencé à préparer cette réinstallation, quand on décida de placer cette bibliothèque dans la pièce supérieure, au $3^{e}$ étage. Elle pourra y être plus facilement agrandie. L'étage sous le dôme lui servira alors de galerie. [...] Le supérieur Trudelle installe lui-même les volumes sur les rayons. Et dans les Annales, il écrit avec une joie visible: "Lorsque cet appartement sera terminé, ce sera le plus beau de la maison". Il y place les nombreuses brochures d'histoire canadienne et de polémique qu'il a soigneusement collectionnées et fait relier, en plusieurs volumes, sous le titre significatif de Musée national. C'est là qu'on voyait les livres par milliers $[. . .]^{51}$. »

Un supérieur comme l'abbé Trudelle, qui assume le rôle du bibliothécaire de son collège, créait assurément une émulation pour la lecture chez les élèves de SainteAnne. On voit le jeune Thomas Chapais qui, à 19 ans en 1877 , détaille les titres de livres qu'il a lus et ceux qu'il souhaite lire pendant son semestre ${ }^{52}$. Le futur journaliste, historien, sénateur et conseiller législatif,

51. Ibid. p. 28-29.

52. Julienne Barnard, Mémoires Chapais, tome 3, Documentation Correspondances - Souvenirs, 1875-1888, Montréal, Fides, 1964, p. 36-38.

\section{Figure 5}

Intérieur de la bibliothèque du Collège à la fin du XIXe siècle, avec au premier plan, les vitrines du musée lapidaire et numismatique ainsi que la maquette du village en 1867 .

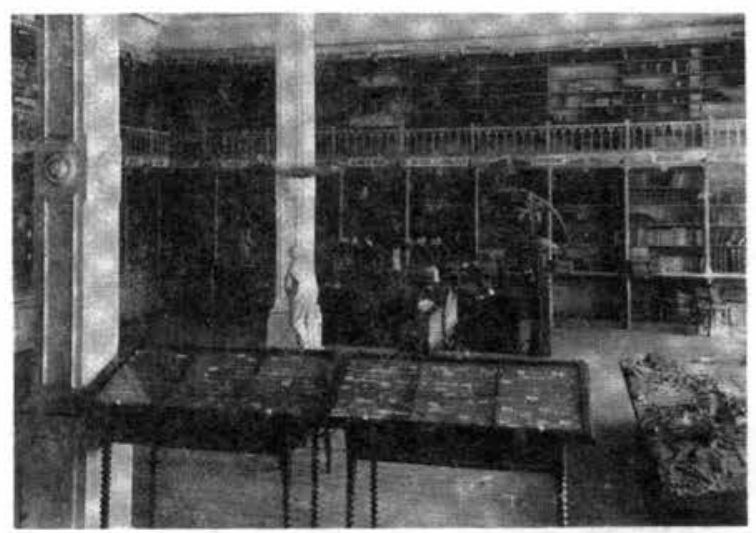

(Archives du Collège de Sainte-Anne-de-la-Pocatière)

issu d'une illustre famille conservatrice de Saint-Denis, lisait entre autres: Joseph de Maistre, Louis Veuillot, Jules Verne, Molière, les écrits d'Oscar Dunn et les conférences de l'abbé Holmes, en plus de biographies, des histoires de France et d'Angleterre.

Une autre activité associée au livre a été greffée à la bibliothèque du Collège. En septembre 1887, l'abbé Charles-A. Collet, directeur des élèves, inaugure la Bibliothèque Saint-Antoine, une fondation de souscription populaire qui fournit des manuels et des classiques aux élèves moins fortunés ${ }^{53}$. Au tournant du siècle, cette bibliothèque de soutien aide ainsi 135 élèves en leur fournissant plus de 700 livres. Ce service s'est poursuivi jusqu'au milieu du $\mathrm{XX}^{\mathrm{e}}$ siècle et atteindra jusqu'à 5000 volumes ${ }^{54}$.

En 1884 , la bibliothèque comptait 14 ooo volumes ${ }^{55}$. Vingt ans plus tard, à l'aube du nouveau siècle, ses collections étaient de l'ordre de 25000 volumes. À partir des années 1880 , les annuaires du collège fournissent le détail des dons faits à la bibliothèque et donnent des renseignements sur son évolution. À défaut d'un catalogue complet des collections à la fin du XIX siècle, les annuaires peuvent combler partiellement la carence des sources.

Au tournant du XXe siècle, 75 ans après sa fondation, le Collège de Sainte-Anne-de-la-Pocatière accueillait environ 1000 étudiants et faisait partie des plus prestigieuses maisons d'enseignement au Québec ${ }^{56}$. L'établissement pouvait afficher un tableau assez remarquable d'anciens élèves ayant fait honneur à leur Alma

\footnotetext{
53. Bibliothèque St-Antoine, ACSCSA, F100/360/8

54. Ibid., p. 134 .

55. ACSCSA, fonds Charles Trudelle, 78 -LII

56. Le Collège dominait la vie d'une communauté qui comptait alors 2500 habitants.
} 


\section{Figure 6}

Le Collège de Sainte-Anne-de-la-Pocatière aujourd'hui.

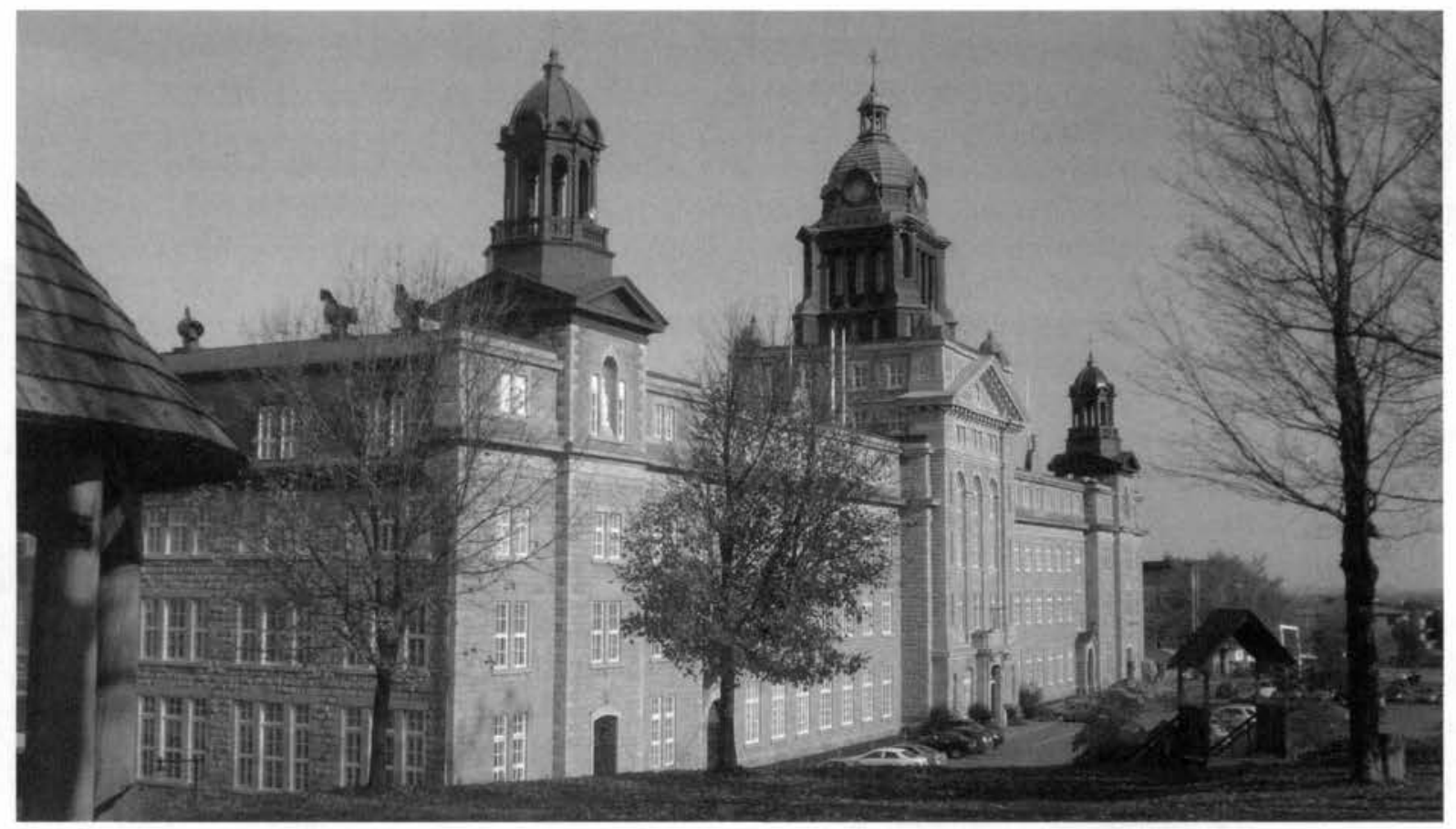

Mater. Si plusieurs prêtres sont sortis du Collège qui privilégiait la formation de séminaristes, Sainte-Anne a aussi formé des médecins, des pharmaciens, des juristes, des enseignants, des journalistes, des arpenteurs, des comptables, des agriculteurs, des industriels, des fonctionnaires, des marins, des architectes, des gens de métiers, etc. ${ }^{57}$ Parmi les plus illustres élèves y ayant complété leur scolarité ou fait une partie de leurs études avant 1900, on peut citer Henri-Raymond Casgrain, dont nous avons parlé, les écrivains Arthur Buies et Louis Fréchette, l'historien et bibliothécaire Narcisse-Eutrope Dionne, le généalogiste Cyprien Tanguay, co-fondateur du Séminaire de Rimouski, les lieutenants-gouverneurs Luc Letellier de Saint-Just, CharlesPantaléon Pelletier, Charles Fitzpatrick et Georges Carroll, les hommes politiques et hommes de lettres Narcisse Faucher de Saint-Maurice, Charles-Ernest Gagnon et Thomas Chapais.

\section{Le Collège et sa bibliothèque aujourd'hui}

En relevant son audacieux défi en 1827 , CharlesFrançois Painchaud a créé une institution qui fêtera bientôt ses 200 ans. Reconstruite à l'égal du collège après l'incendie de 1920, sa bibliothèque s'est modernisée et accueille fidèlement de nouvelles cohortes d'élèves qui fréquentent ses rayons et empruntent encore ses documents, comme leurs lointains confrères du $\mathrm{XIX}^{e}$ :siècle. Les temps ont changé, mais l'instruction et la lecture demeurent pour conjuguer les souvenirs aux espérances et les apprentissages aux projets.

En 1827 , on lisait le soir à la lueur des chandelles; au $\mathrm{XXI}^{\mathrm{e}}$ siècle, la lumière des écrans voisine les livres imprimés et, comme les anciens élèves, ceux d'aujourd'hui découvrent des œuvres et des savoirs qu'ils ne connaissaient pas avant d'arriver au Collège. La bibliothèque éclaire le maître autant que l'élève, tisse des liens dans le temps et permet au passé d'enrichir l'avenir. Depuis près de deux siècles, sur son remarquable promontoire face au Saint-Laurent, le Collège de Sainte-Anne-de-la-Pocatière fait toujours face au vent du large et sa bibliothèque déploie toujours l'horizon des esprits. (-)

57. Pour un tableau statistique complet des professions des élèves du Collège de La Pocatière au XIX ${ }^{e}$ siècle, voir: Catalogue des anciens élèves du Collège... op. cit., p. 414. 